\title{
Constraint-Based Support for Collaboration in Design and Construction
}

\author{
Claudio Lottaz*, Denis E. Clément**, \\ Boi V. Faltings* and Ian F.C. Smith** M.ASCE \\ * Computer-Science Department \\ ** Department of Civil Engineering \\ EPFL - Swiss Federal Institute of Technology \\ CH-1015 Lausanne, Switzerland
}

April 3, 2000

\begin{abstract}
Solution spaces are proposed, instead of single solutions only, to support collaborative tasks during design and construction. Currently, partners involved in construction projects typically assign single values for sub-sets of variables and then proceed, often after tedious negotiations with other partners, to integrate these partial solutions into more complete project descriptions. We suggest the use of constraint solving to express possibly large families of acceptable solutions in order to facilitate and abbreviate the negotiation process. New algorithms have been developed at EPFL for solving multi-dimensional non-linear inequality constraints on continuous variables. Constraint-based support leads to better change management and easier implementation of least commitment decision strategies. It is expected that the results of this research will improve the performance of negotiation systems currently in use.
\end{abstract}

\section{Introduction}

Most engineering tasks require collaboration between many partners. Collaboration tasks are complicated by factors such as losses during information exchange, misunderstandings and iterative negotiation when sub-task solutions conflict. Moreover, changes in context, costs, requirements, deadlines, etc. require constant renegotiation of issues that may modify important project characteristics.

Although communication and collaboration tools recently became more attractive with the success of the Internet, little work proposes support for maintaining project consistency. For example, many projects (e.g. $[4,7,11,32,37,31]$ ) contain proposals for communication and information-management facilities such as graphical data bases, shared project models, knowledge bases, bulletin boards and video conferencing. Much of this work is founded on the assumption that improved communication helps engineers carry out tasks such as negotiation. 
Inconsistent construction projects are costly and therefore, there has been much research into explicit computer support for negotiation and conflict mitigation. Beginning with work into design rationale [26], Peña-Mora has recently proposed a combination of negotiation and game theory to support negotiation between partners [27, 28]. Ndumu [25] suggests a computational market model to resolve conflicts. Scherer [36] has drawn from research into product modelling, work-flow management, logistics and artificial intelligence to create a framework that is expected to reduce the time before construction by $50 \%$. Garcia and Vivacqua [12] propose the use of active design documents to acquire and retrieve design rationale while conflicts are under negotiation. Mokhtar et al [24] focus on change management to provide an information model that assists in planning and scheduling design changes. None of this work proposes constraint solving for negotiation support. If constraints are used, they are employed to perform consistency checks; there is no constraint propagation and no proposal to employ constraints for determining solution spaces.

When constraint solving is used, only local consistency is usually assured. Bahler et al $[1,3]$ proposed a design advice tool that uses constraints to support negotiation and conflict resolution. Since only arc-consistency is maintained, this system is reliable only when the constraint network has the form of a tree graph. An exception handling approach studied by Klein [18] also uses local methods for enhancing consistency. Most constraint networks contain cycles; this problem was originally identified as a challenge to collaborative civil engineering support by Sriram [42]. Realizing the need for constraint solving in the Redux system [29, 30] Petrie has enhanced the framework to include a constraint manager that would plug into remote solvers. Khedro and Genesereth [17] have developed a progressive negotiation strategy for conflict resolution where locally consistent solutions are used to converge on a global consistency. However in these studies, no explicit use of solution spaces has been found for constraints expressed in terms of continuous variables.

Ivezic and Garrett [15] employ constraints which are not available as explicit relationships but which are deduced from simulation and probabilistic behavior models. Simulation data is used to train neural networks. The Monte Carlo method is applied on these neural networks in order to generate statistics. Users then interactively narrow valid ranges of form and behavior variables.

Currently in practice, much negotiation effort can be traced to partners that determine only one solution for individual subtasks. Integration of partial solutions often leads to artificial conflicts. Such conflicts may be less related to conflicting project goals than to premature decision making. Decision making is often required only to specify single solutions that demonstrate satisfaction of subtask requirements.

Constraints provide a useful format for representing solution spaces. Constraint satisfaction techniques compute approximations of solution spaces of constraint satisfaction problems (CSPs). A CSP is defined by a set of variables, their domains (a priori possible values for each variable) and a set of constraints. In engineering, constraints are typically numerical relationships (equalities and inequalities) that influence feasible values of continuous and discrete variables. This paper provides support for solving constraints expressed in terms of continuous variables.

The task of the CSP is to identify variable values that satisfy its constraints and that are within allowable variable domains. A measure of success when carrying out this task is called consistency. In general, CSP algorithms that ensure low degrees of consistency over-estimate the solution space but typically have low computational complexity. CSP algorithms that ensure high degrees of consistency provide a tight estimation of the solution spaces but suffer 
from high complexity. Complexity is defined in the computer science sense, thereby referring to the sensitivity of execution time to the amount of input data.

If collaborating partners specify CSPs instead of single solutions to their subtasks, solutions to the complete project task are obtained through solving the CSP formed by the conjunction of sub-CSPs. Solution spaces defined by CSPs have the potential to improve efficiency in collaboration. In addition, better solutions would emerge because project partners were less restrictive when confronted with innovative ideas. A key challenge to the adaptation of CSPs in collaborative engineering is the difficulty associated with solving very large CSPs on continuous variables.

CSPs have been already proposed to describe solution spaces by Darr and Birmingham in the system ACDS [5]. A distributed agent architecture solves collaborative tasks using catalogues and restrictions instead of single propositions by collaborators. Component catalogues and requiremnts specified on parameters are considered as a CSP on continuous variables. Domains of the variables are deduced from the catalogues and the requirements collected from collaborators. After imposing local consistency on parameter ranges, many components can be eliminated before starting the search for the best solution using utility functions. The combinatorial explosion during search is thus weakened.

This paper begins with an outline of the advantages of constraint satisfaction and solving algorithms for representing solution spaces. After a short introduction to constraint satisfaction techniques, an Internet-based implementation called SpaceSolver is described. Constraints on continuous variables may assume any analytical form. Two examples in steel construction are used to illustrate the use of of this tool and finally, current limitations and opportunities for future work are discussed within the context of the construction industry.

\section{Collaboration through constraint solving}

When data transmission is augmented by explicit project constraints, collaboration systems can provide much additional engineering support. More specifically, representation, exchange and manipulation of constraints have the following advantages :

- a description of solution spaces is available

- artificial conflicts are avoided

- the initiator of a change retains responsibility for maintaining consistency

- when changes in requirements occur, much work can be reused

- negotiation is guided within feasible spaces

- optimally directed decision making is bounded

Most of the previous work in this area can be enhanced through realization of one or more of these advantages. More details and an illustrative example are given below.

A description of solution spaces is available CSPs - especially those having constraints in terms of sets of values for discrete variables and inequalities of continuous variables - offer the possibility to calculate, represent and manupulate solution spaces. Through departing from traditional point solutions (one value for each variable), CSPs augment the 
amount of information available for subsequent decisions. For example, CADRE [14] and IDIOM [41] use constraint solving on constraints on geometric parameters to enhance appartment floor layout plans, thereby facilitating adaptation.

Use of CSPs help delay decisions for variable values until they become essential for the completion of the project. When premature decsions are reduced, information related to possible alternatives is retained. This is a variant of the least commitment paradigm often employed for planning tasks. In the automotive industry, decision delay strategies have already been adopted by major manufacturers, for example [45].

Artificial conflicts are avoided Many conflicts in traditional collaboration arise from forced early decisions related to the values of variables. Often, there is no real conflict even though a negotiated change between two partners cannot converge to an acceptable solution for all partners. When negotiating over single values for parameters, collaborators provoke artificial conflicts that lead to needless iterations of negotiation.

For example, Table 1 illustrates the evolution of four values for variables as each project partner applied constraints related to their goals for the task. This example refers to geometrical parameters of a beam with holes for passing ventilation ducts. Values $d$ are hole diameter, $e$ is the hole spacing (center to center), $h$ is the height of the beam, and $x$ is the distance to the first hole. It was inspired from the design, fabrication and erection of a steelframed building in Geneva, Switzerland. More details are provided in Section 5. Each row represents an iteration; the partner named in the row initiates the change and bold values are the changed values.

\begin{tabular}{|l|cccc|}
\hline & $d$ & $e$ & $h$ & $x$ \\
\hline Architect & 550 & 650 & 650 & 500 \\
Steel fabricator & 550 & $\mathbf{9 0 0}$ & 650 & $\mathbf{1 1 0 0}$ \\
Engineer & $\mathbf{2 0 0}$ & 900 & 650 & 1100 \\
Architect & 200 & 900 & 650 & $\mathbf{1 0 0 0}$ \\
Ventilation sub. & $\mathbf{4 5 0}$ & $\mathbf{8 0 0}$ & 650 & $\mathbf{6 0 0}$ \\
Engineer & $\mathbf{4 0 0}$ & $\mathbf{9 0 0}$ & $\mathbf{7 3 0}$ & $\mathbf{7 0 0}$ \\
Steel fabricator & 400 & 900 & 730 & $\mathbf{8 0 0}$ \\
\multicolumn{1}{|c|}{$\vdots$} & $\vdots$ & $\vdots$ & $\vdots$ & $\vdots$ \\
\hline
\end{tabular}

Table 1: Needless negotiation iterations. This example was inspired from a real case in steel construction. Numbers are in $\mathrm{mm}$.

In Table 1 it is not clear that these iterations will ever converge on values that are acceptable to all partners. In reality, the steel contractor had to assume values before these iterations had terminated in order to satisfy the construction schedule. Several thousand dollars were wasted because the assumed values were eventually refused by another partner.

In this case, there is a large space of solutions that would have been acceptable to all partners. When solution spaces are considered, any subtask allows calculation of a local solution space for common variables. The intersection of all local solution spaces contains the possibilities for the whole task, see Figure 1

When allowable values for the whole task are computed, it becomes possible to visualize solution spaces for selected parameters, see Figure 2. Simple unary and binary constraints were used to approximate the solution space for a constant beam depth of $700 \mathrm{~mm}$. Had this type of CSP solution been available to the partners involved in the steel-framed building, 


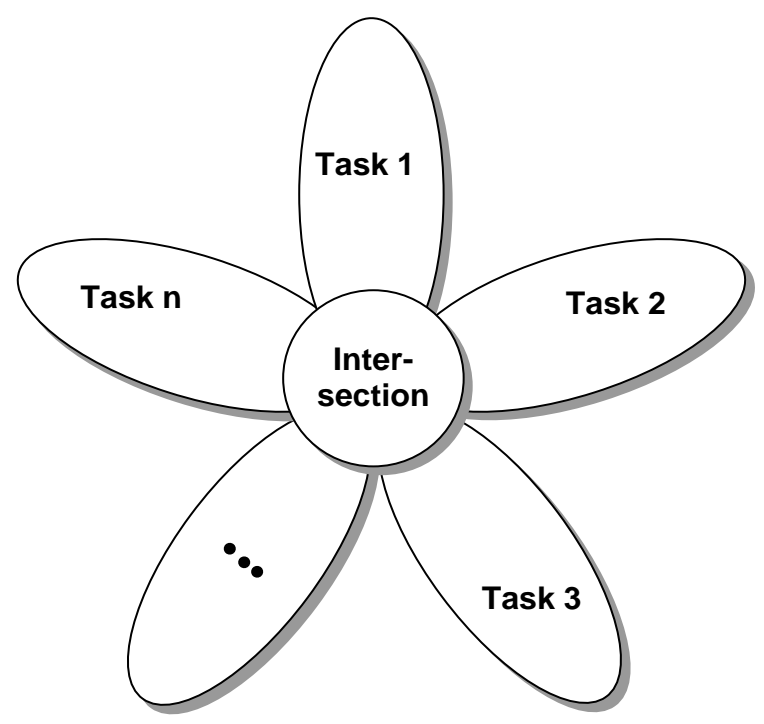

Figure 1: Subtasks define a set of values on common variables in local solution spaces. The intersection of these spaces is the solution space for the whole problem.

no iterative negotiation would have been necessary. More details, including more accurate constraints on these variables, are provided in Section 5.

The initiator of a change retains responsibility for maintaining consistency. In spite of the advantages of using solution spaces and delaying commitment to variable values, it is often necessary to work with point solutions (such as drawings). Values for parameters of such solutions change frequently over the life of a project. Early collaboration systems failed because responsibility shifted to the receiver of the change. If there is a shift in responsibility partners lose control of the project when they become unable to maintain consistency. The result is a project that is more expensive, has lower overall quality and takes longer to complete than a project without any computer support at all. Collaboration systems can make matters worse.

When solution spaces are calculated, the initiator of a given change to a point solution has information related to consistent values before the change is decided upon and communicated to others. Therefore, it becomes possible to require, for example, that all modifications should fit into the declared solution space in order to avoid initiation of a special negotiation procedure. In this way, constraint methods and the subsequent solution space representation are able to forewarn the change initiator of consistency problems and subsequent extra work if changes are not within solution spaces. As a result, recipients of changes (who are often civil engineers) are much less likely to be overwhelmed by verification tasks as the project progresses.

Better management of changes in requirements Changes in requirements within traditional negotiation systems often mean that much negotiation must be revisited. When a constraint-based approach is used, changes to requirements lead to the introduction of new constraints and modifications to existing constraints. Recalculation of solution spaces is then done automatically and this supports better change management.

Negotiation is guided within feasible spaces Negotiation is unavoidable. A solution space is a representation of the space of feasible alternatives and knowledge of its shape 


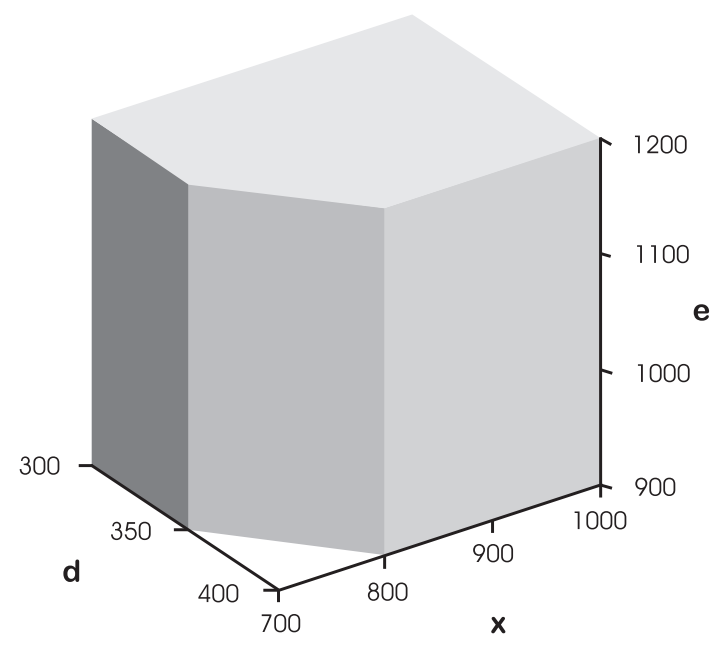

Figure 2: Calculation of solution spaces provides the opportunity to produce graphical representations of acceptable values for parameters. Values are in $\mathrm{mm}$.

usually encourages partners to be less restrictive. Therefore, solution spaces provide useful support for negotiation.

Beyond the solution space, project partners become aware that variable value changes will also require modification to project constraints. The edges of solution spaces thus represent boundaries between different types of negotiation. Also, the structure of a CSP may assist in determining the order in which inconsistencies should be resolved. Knowledge of the type of negotiation and the importance of the inconsistency helps schedule negotiation activities.

Optimally directed decision making is bounded In civil engineering, modelling of complete project knowledge is rarely feasible and therefore, optimization is an unattainable goal. However within solution spaces, it may be useful to provide support for identifying solutions that are better according to selected criteria. This is called optimally directed decision making and many algorithms exist, for example [30], to support such effort. Solution spaces improve the efficiency of these algorithms through defining the sets of possible point solutions.

\section{Constraint Satisfaction Techniques}

Much civil engineering knowledge is expressed in terms of constraints. Building codes, design criteria, behavior models, cost restrictions and planning strategies all employ explicit declarations of constraints. Computer systems that propose support for civil engineering tasks often involve a transformation of this knowledge into other forms such as rules, directed relationships and post facto tests. Such transformations reduce clarity for engineers who use such systems and this results in systems that are hard to maintain and often incomprehensible to use. Since much engineering knowledge is already in constraint format, direct use of constraint representations is obviously desirable. Constraint-based systems have the potential to be one of the most understandable and the easiest to maintain of all reasoning systems proposed for many engineering tasks. 


\subsection{Definitions in constraint satisfaction}

Constraint problems are generally formalized in terms of variables which represent properties of components and constraints which specify the restrictions that must hold for any valid solution of the task. Sets of constraints and variables together with their allowable values (domains) are called constraint satisfaction problems (CSPs). CSPs are generally represented using graphs (or hyper-graphs) called constraint networks. In such graphs, nodes are usually variables and arcs are constraints between variables. A solution to a CSP is an instantiation of all variables such that all constraints are satisfied simultanuously. A solution space of a CSP contains all solutions of the given CSP.

CSP algorithms fall into two categories. Consistency techniques use a variety of methods to restrict the search space to interesting regions. They prune parts of the search space to discard those branches where no solutions are expected. The second category includes splitting and backtracking techniques for searching for solutions. The task of solving CSPs in their general form is NP complete.

One important class of CSPs is those defined for variables that have finite domains. Efficient methods to achieve various degrees of consistency [2, 22, 23] are available and sophisticated techniques for identifying solutions through intelligent backtracking exist [19]. While in many fields such as planning and resource allocation it is often useful and efficient to consider variables with finite domains only, tasks in engineering employ variables that have continuous values.

\subsection{Finding single solutions for continuous CSPs}

Resolution of CSPs involving variables with continuous domains (CCSPs) is different from solving CSPs on variables with finite domains. Most successful resolution techniques for numerical constraint satisfaction problems on continuous variables concentrate on finding single solutions, possibly considering optimization criteria. Most techniques are not related to traditional CSP research. Popular techniques include linear and non-linear programming, numerical analysis, hill-climbing and stochastic techniques. Such approaches are adequate when a problem is entirely specified; complete specification makes it reasonable to search automatically for the best solution although many solutions may exist. However, since complete specification cannot feasibly be achieved in construction, more sophisticated support for decision-making is desirable. Recent work on continuous CSPs provides techniques for approximating solution spaces using intervals $[8,13,20]$ and some work has proposed methods for accurately calculating solution spaces $[33,34]$.

\subsection{Local Consistency}

Local consistency is an approach for approximating solution spaces. The original searchspace of a CSP is defined by the cross-product of the domains of all variables. Local consistency algorithms prune parts of this search-space using local inconsistencies. Such inconsistencies are incompatibilities between values of closely related variables.

There are many ways to achieve local consistency and each method provides a different degree of reliability [8]. One can distinguish different orders of consistency: 1-, 2- and in general k-consistency, depending on the degree, $k$, of locality taken into consideration. A k-consistency algorithm furnishes an approximation of the solution space where each subproblem of $k$ variables is guaranteed to be consistent. This amounts to ensuring that each 
partial solution of $\mathrm{k}-1$ variables can be extended consistently to a partial instantiation of $\mathrm{k}$ variables.

In practice, this is achieved by assigning a label to each subset of $\mathrm{k}-1$ variables which contains their legal value assignments. Thus a 3-consistency algorithm is supposed to calculate a two-dimensional label for any pair of variables. For any combination of values contained within such a label, any third variable can be instantiated such that all constraints of the CSP still hold.

Although k-consistency ensures extensibility of partial solutions involving $\mathrm{k}-1$ variable to any $k$-th variable, there is in general no guarantee that such partial solutions are extensible to a complete solution if the CSP contains more than $\mathrm{k}$ variables. Usually locally consistent labelings overestimate the solution space and it is even possible that a locally consistent label does not contain any value combination which is extensible to a complete solution. However, if a local consistency algorithm results in an empty labeling, it is sure that no solution to the corresponding CSP exists. As a result, local consistency methods are often used in practice to improve search through pruning inconsistent values from the domains of the variables and to detect empty solutions.

\subsection{Global Consistency}

Construction projects require that all values for variables are globally consistent with project constraints. Inconsistencies cause project delays, extra costs and accidents. Therefore, a stronger notion than local consistency is desirable.

When the labeling constructed by a consistency algorithm contains only those values or value combination which occur in at least one solution, it is said to be globally consistent. A globally consistent labeling is a compact and conservative representation of all solutions admitted by a CSP. It is sound in the sense that the labeling never admits any value which does not lead to a solution. It is complete in the sense that all solutions are represented in it.

In general, a globally consistent labeling may require explicitly representing constraints for all variables in the problem (i.e. computing n-1-dimensional labels for a problem of size n), a task which has exponential time complexity in the worst case. Recent results show, however, that for special classes of problems, low orders of consistency are equivalent to global consistency. These results lead to polynomial time algorithms for computing globally consistent labelings and can be summarized as follows:

- 2-consistency (also called arc-consistency) is equivalent to global consistency when the constraint network is a tree graph [10],

- 3-consistency (also called path-consistency) is equivalent to global consistency when the CSP is convex and binary [6, 43, 34]. A CSP is convex when all its constraints are convex. A constraint is convex if the straight line between any two feasible points entirely lies within the feasible region. A CSP is binary if constraints involve at most two variables.

- 3,2-relational-consistency (a variant of 5-consistency defined in [33, 34]) is equivalent to global consistency when the CSP is convex and ternary. A CSP is ternary if any 
constraint contains at most three variables. Note that a numerical CSP, stated analytically, can always be transformed into a ternary one without loss of information using syntactic transformations.

Thus algorithms which acheive 2-, 3- and 3,2-relational consistency can compute complete and sound descriptions of the entire solution space at low computational cost under certain conditions. When the problem has no special simplifying conditions, these algorithms are often useful pre-processing tools for reducing the complexity of the search space. They could then be interleaved with interval-based backtrack-search algorithms to generate consistent sub-regions of the solution space [16]. Alternatively, users may wish to explore the solution space interactively, particularly when it is known that the formalized knowledge is incomplete.

Figure 3 illustrates the different types of approximations provided by arc, path and 3,2relational consistency respectively. It shows projections of the arc, path and 3,2 relational consistent spaces onto the same three variables. Since the representation of an arc-consistent space is a collection of unary labels, its projection onto three variables is a cube or in general a set of cubes (Figure 3a). The same projection of a path-consistent space is the intersection of three prisms, since it is represented using two dimensional labels (Figure $3 \mathrm{~b}$ ). 3,2-relational consistency uses three dimensional labels and therefore general forms in 3dprojections (Figure 3c). This problem involves 16 variables and 12 constraints. Any three variables can be displayed once label consistency has been acheived.

\section{An Internet-based Collaboration Tool}

SpaceSolver is an Internet-based software package that provides consistency techniques for numerical continuous CSPs. It also contains many features that are useful in collaboration, such as data-management and protection tasks for shared and private data for collaborative projects. It is available at the URL http://liawww.epfl.ch/〜ottaz/SpaceSolver/.

The platform uses a complex tree-graph representation of continuous constraints for computing a labeling of any degree of consistency in continuous domains. This data-structure was originally developed in computer vision [35] and was later proposed for engineering design in $[33,34]$ This representation is based on the assumptions that i) in most practical applications each variable takes its values in a bounded domain (bounded interval) and ii) there exists a maximum precision with which results can be used. Provided that these two assumptions hold, a relation can be approximated by carrying out a hierarchical binary decomposition of its solution space into quadtrees for binary relations and octrees for ternary ones (see Figure 4). Graphically in two dimensions, this means that rectangles that are not completely in the solution space are subdivided into four smaller rectangles and re-tested iteratively until the desired precision is obtained.

Numerical constraints generally arise as algebraic or transcendental equalities and inequalities involving several variables. Traditional CSP approaches process constraints directly in this form and therefore they encounter analytical difficulties related to intersecting surfaces and finding extrema. This partly explains why the most prominent advances in numerical constraint satisfaction are related to 2-consistency [8, 13, 20, 44].

Instead of an implicit representation of numerical constraints, the quad/octree representation leads to a logical rather than numerical treatment of continuous solution spaces. 


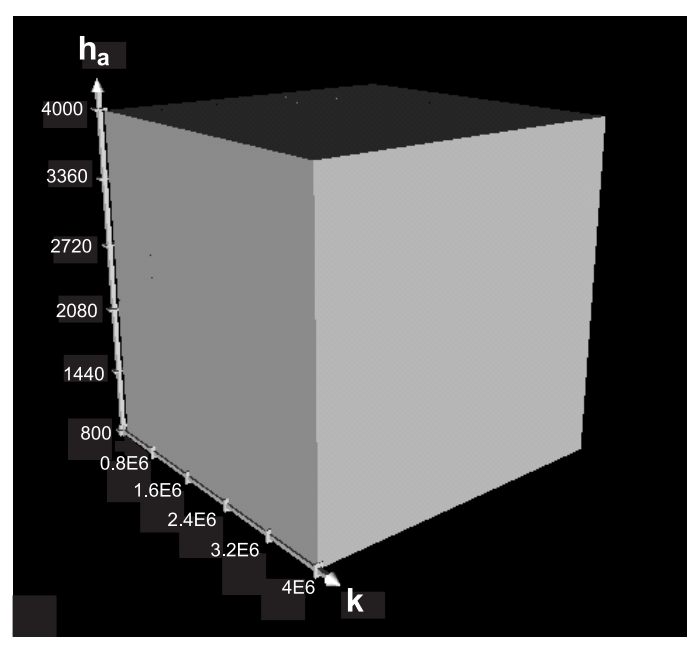

a)

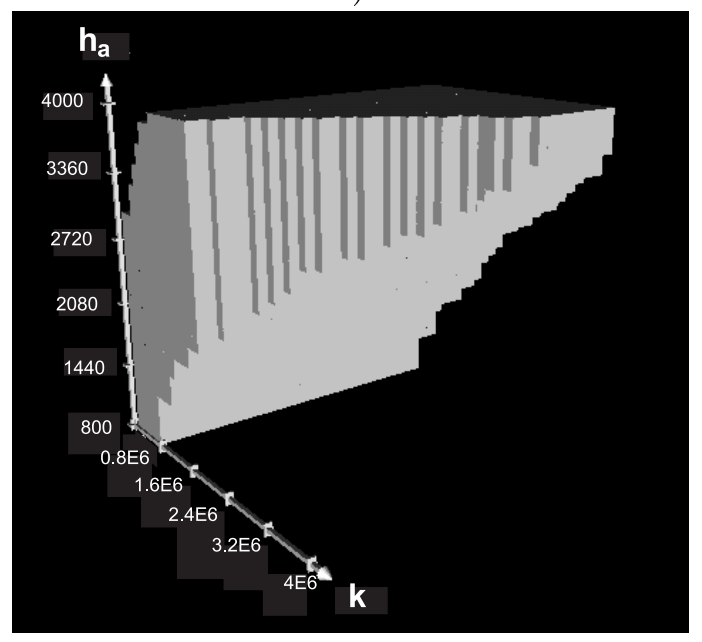

b)

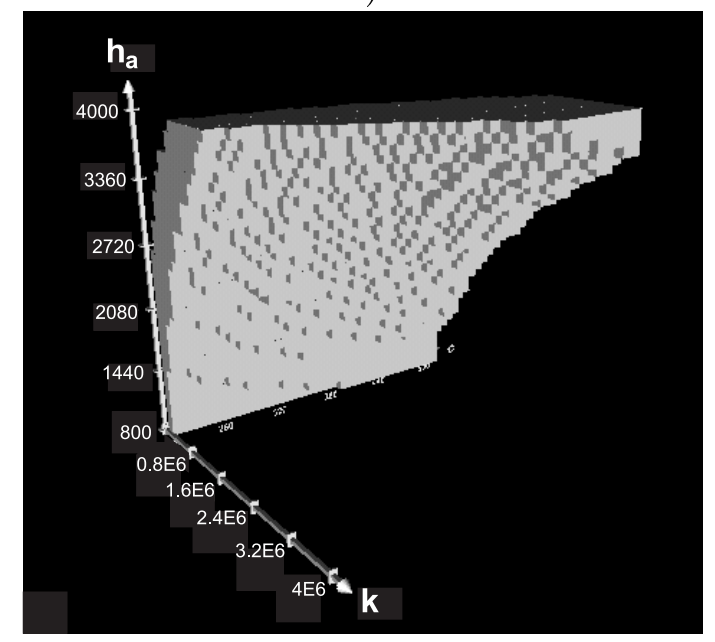

c)

Figure 3: Consistency techniques approximate the solution space of CSPs. This figure shows 3d-projections onto the same variables of a) the arc-, b) the path-, and c) the 3,2-relational consistent space of the same problem. This solution space was calculated using the information from Application 2, Section 5.4. The solution space projection for any three variables in the constraint network can be shown. 


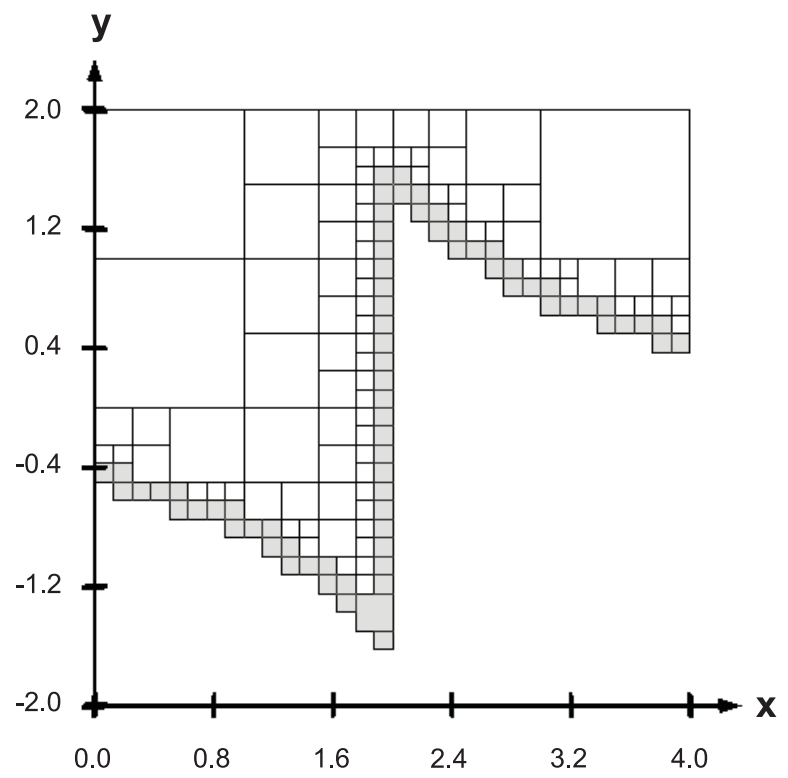

Figure 4: A continuous relation is approximated by carrying out a hierarchical binary decomposition of its solution space into a $2^{k}$-tree graph. In this figure $k=2$ and therefore, each subdivided rectangle that is not completely in the solution space results in four smaller rectangles. This figure shows $y \geq \arctan \left(\frac{1}{x-2}\right)$.

This allows for a simple implementation even for high degrees of consistency in continuous domains (see [33] for further details of the quad/octree construction and consistency algorithms).

\subsection{System Architecture}

SpaceSolver is developed as an Internet application, in order to make consistency techniques on continuous variables available world-wide. The system architecture retains most modules on the server, thus avoiding client-side plug-ins or Java-Applets and thus making the solver more independent of the user's machine and configuration. The disadvantage of this approach is that the server can become overloaded when several projects are being treated at once. However in the near future, improvements in server capacity are likely to have a greater effect on this sort of communication than efforts to harmonize all possible client machines and configurations.

Figure 5 illustrates SpaceSolver's system architecture. On the client side any JavaScript-enabled HTML-browser communicates through standard Internet protocols with the WWW-server where SpaceSolver is installed. Whenever a user is manipulating CSPs, asking for calculation or visualising constraints and solution spaces, the WWW-server calls the corresponding CGI-scripts to perform the demanded operation. These scripts are written in PERL and communicate through UNIX-sockets with the SpaceSolver-server. The SpaceSolver-server has the rights to perform activities such as writing files containing CSP-definitions and executing SpaceSolver-modules. These modules include the symbolic manipulator, the constraint converter, the constraint solver, and the VRML-Generator.

Several scripts for Maple V are used for the Symbolic Manipulator which eliminates unnecessary auxiliary variables and transforms the CSPs into ternary form. The Constraint 


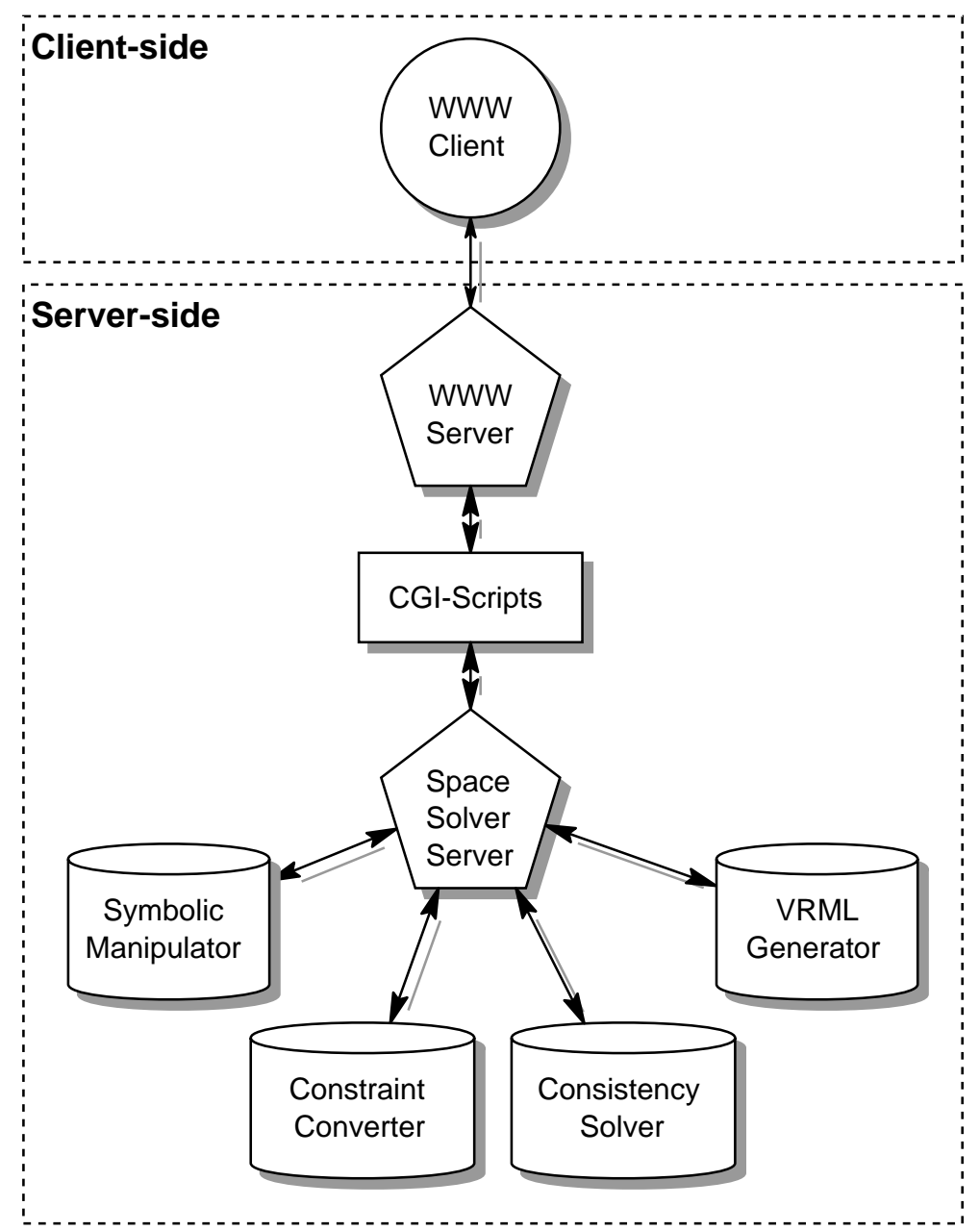

Figure 5: SpaceSolver's architecture does not require users to install plug-ins and run java-applets through carrying out most tasks on the server. 
Converter is used to convert constraints from symbolic form into an explicit spatial representation. The Constraint Solver module implements consistency algorithms such as arc-, path- and 3,2-relational consistency. To achieve best performance, this module and the constraint converter are written in $\mathrm{C}++$. Finally, the VRML-Generator dynamically generates 3D VRML models of constraints and solution spaces in order to view constraints and solution-spaces on the Internet.

\subsection{WWW-Interface}

When users connect to the SpaceSolver-WWW-page, they are prompted for a user-name and a password. This user authentication allows us to maintain separation of data and to control access to our projects. Registration is free.

When users select an existing CSP network or create a new one, they are presented a page similar to Figure 6. On this page, users specify equalities and inequalities in ASCII-text using Maple V's syntax, as well as minima, maxima, default values and short description for each variable. The table that specifies variables, shown in the lower part of Figure 6 , is generated automatically by detecting the variables found in the constraints.

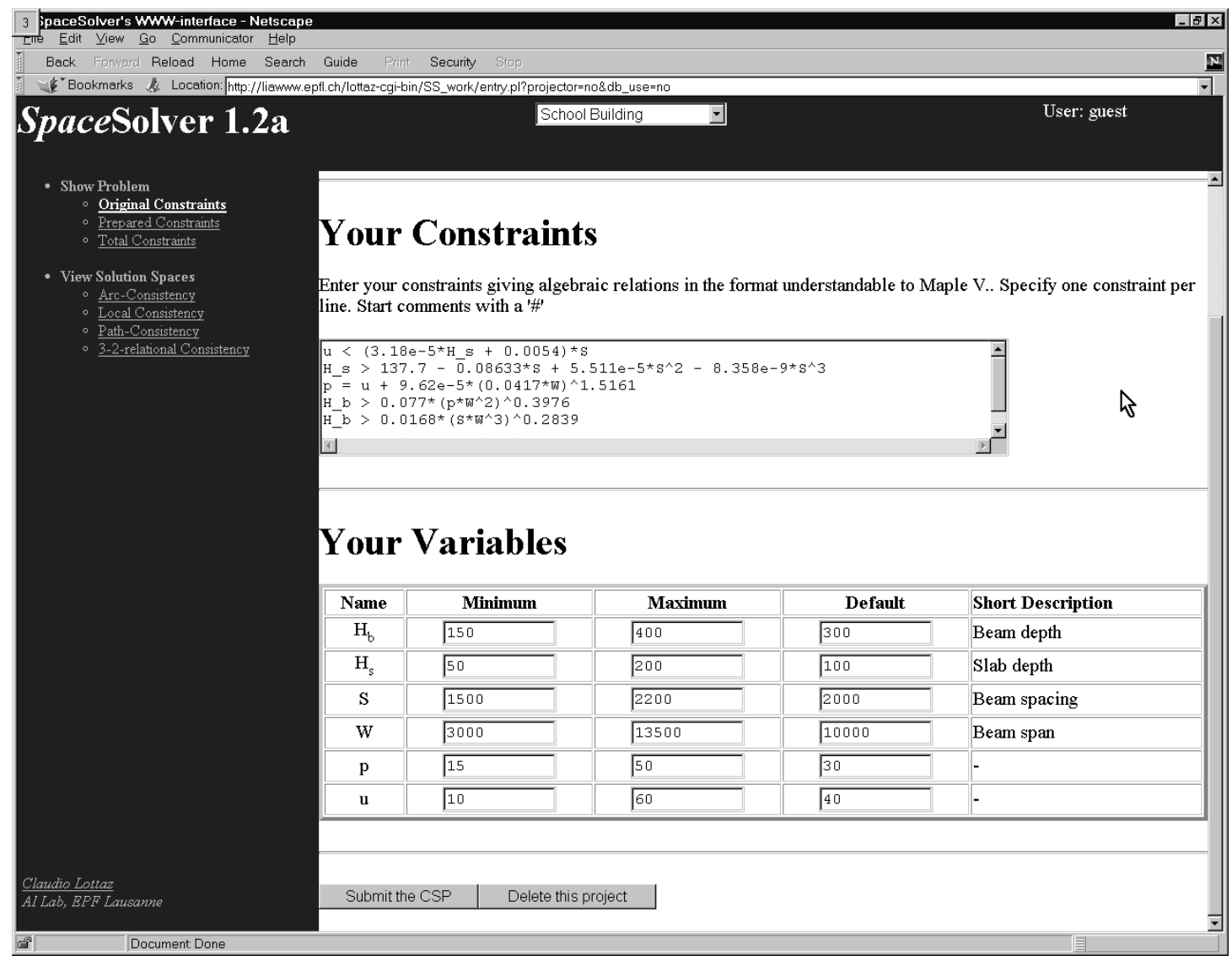

Figure 6: SpaceSolver's WWW-Interface allows specification of CSPs in an intuitive way through the Internet

For collaborative work SpaceSolver provides the possibility for several users to partici- 
pate in the same project. Collaborators specify their personal constraints on partially shared variables. The domains of shared variables can be influenced by all partners who use it. Collaborators may be added to and removed from a project by its creator. In order to facilitate discussions and explanations, SpaceSolver is linked to the ICCS-database, a collaboration environment for construction [38].

Visualisation of constraints and solution spaces helps obtain an understanding of characteristics of the constraint network and this supports decision making. Suppose, for instance, that an engineering task has three (partial) optimisation criteria. Through visualising the projection of the solution space on these criteria, trade-offs may be illustrated and possible alternatives can thus be examined. In order to provide visualisation, VRML-scenes representing constraints and solution spaces are generated dynamically (see Figure 7). VRML is a 3D modelling language for the Internet. Several plug-ins to WWW-browser and standalone VRML-browsers allow Internet-users to examine scenes specified as VRML. Projections of the solution space on any triplet of variables can be generated and visualized in Space Solver.

\subsection{Symbolic Pre-Treatment}

Although SpaceSolver's consistency techniques are based on spatial representations of constraints and solution spaces, it performs symbolic pre-treatment for two reasons. First, the number of variables involved in the CSP is important for the efficiency of the consistency algorithms. Therefore avoidable auxiliary variables should be eliminated. Second, the consistency algorithms described in Section 3 treat CSPs in ternary form, i.e., each constraint must involve at most three variables. Thus SpaceSolver substitutes constants and auxiliary variables defined by functional equations, and makes CSPs ternary.

Substituting constants: Equalities of the form $v=$ const are used to substitute $v$ by const in the whole CSP. This kind of equality may occur when designers commit to a value for a parameter, or to make the CSP-specification more readable.

Substituting auxiliary variables: Certain auxiliary variables are defined as an expression of only one other variable $x$, for example, $v=\operatorname{expr}(x)$. Since this dependency assigns to each value of $x$ exactly one value of $v, v$ is substituted by $\operatorname{expr}(x)$ in the whole CSP. Variables can thus be eliminated without increasing arities of constraints.

Making CSPs ternary: In general CSPs contain constraints which involve an arbitrary number of variables. For numeric CSPs, however, it is possible to introduce auxiliary variables such that all constraints are expressed using ternary constraints. The brute force method that involves introducing an auxiliary variable for each intermediate result generates many auxiliary variables. Therefore we use a more sophisticated algorithm that introduces as few auxiliary variables as possible through reusing expressions as often as possible.

\subsection{Consistency Algorithms}

SpaceSolver implements the consistency concepts introduced in Section 3. Two algorithms for arc-consistency are available: the standard version [33] and an improved one inspired by [9]. The original path- and 3,2-relational consistency algorithms have been substantially improved by avoiding the storage of large intermediate five-dimensional data structures. This improvement can reduce memory needs by a factor of ten. 
Constraints involving the same set of parameters are stored in one total constraint. The original constraints are intersected to form one single constraint, thereby simplifying the CSP. A space enclosed by this constraint is shown in Figure 7.

\section{Applications}

\subsection{Application 1: HVAC in a steel framed building}

The example is inspired by an existing building in Geneva, Switzerland. Poor collaboration resulted in higher than necessary construction costs. In buildings that house various sizes of servers and other large computers, ventilation requirements are important design criteria. Good ventilation maintains satisfactory operating temperatures and this leads to greater equipment reliability. Therefore, space has to be reserved for ventilation ducts. However, building height cannot be increased without having an impact on construction and operating costs.

Beams with holes allow passage ventilation ducts where beam material is not used efficiently, thereby providing effective solutions at reasonable costs. The choice of number of holes and ducts, spacing of the beams, beam height, hole diameter and other geometric parameters is however not easily established. Typically, this issue generates much discussion and negotiation between collaborators.

The project collaborators that are most concerned with this issue are architects, engineers, the steel fabricator and ventilation subcontractors. Architects aim for an aesthetically pleasing distribution of holes and good proportions of hole size with respect to other dimensions. Engineers typically require a few small diameter holes so that beam strength is not compromised. The steel fabricator prefers high values for hole spacing and no hole proximity to connections in order to avoid effects of stress concentrations caused by the holes. Finally, ventilation subcontractors want large, closely spaced holes everywhere so that they can accommodate later changes most easily. Such conflicting goals are common in every construction project.

\subsection{Describing the problem with constraints}

Use of constraints requires clearly defining the relevant variables and their relationships. Figures and tables help to describe variables and avoid confusion between disciplines which have different definitions for variables. For example, the variable "A" is used to symbolise area : engineers used it for chord area; the ventilation subcontractors use A for duct area; and the architects employed A for floor area. Maintaining non conflicting terminology is an essential and continuous task.

Next, partners introduce their constraints, constants and variables along with their range of acceptable values. The steel fabricator, for example, introduces a variable that represents the distance between the first hole and the column supports and states that this value should be greater than 1.5 times the hole size. Also, this value must be more that $250 \mathrm{~mm}$ and less than half the span of the beam. Other values, domains and constraints related to manufacturing, assemblage and transport are also introduced.

The ventilation subcontractor expresses the hole diameter $(d)$ needed in terms of the number of ducts $\left(n_{d}\right)$, considering insulation features $\left(d_{o}\right)$ and the comfort (air renewal $\left(c_{v}\right)$ required by the owner. The maximum air speed $\left(F_{r}\right)$ can be set in order to specify appropriate 


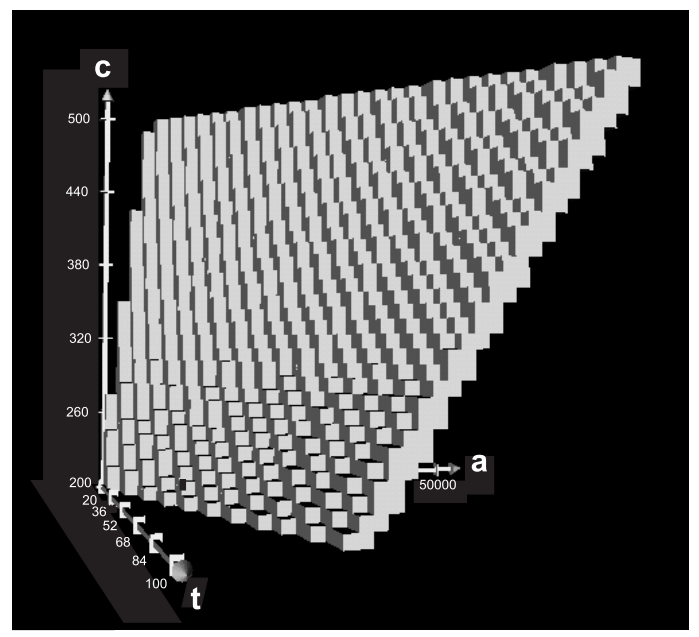

a): $a=c t$

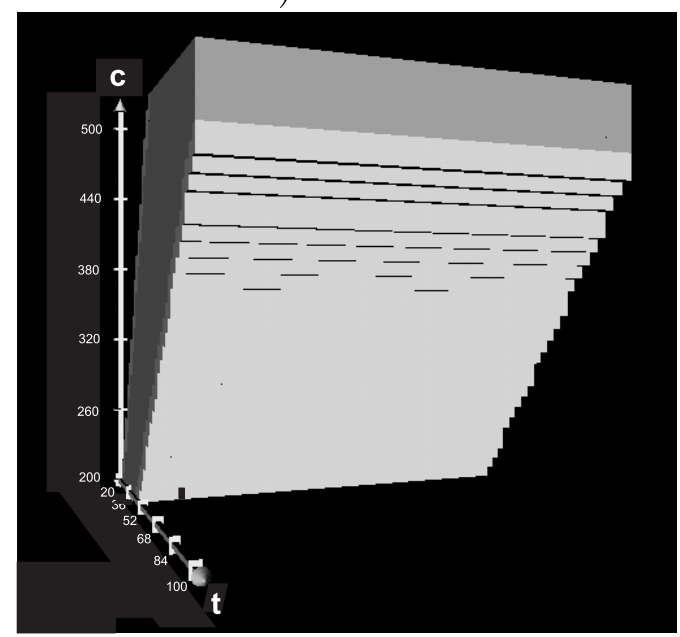

b): $4.5 t \leq c \leq 8.5 t$

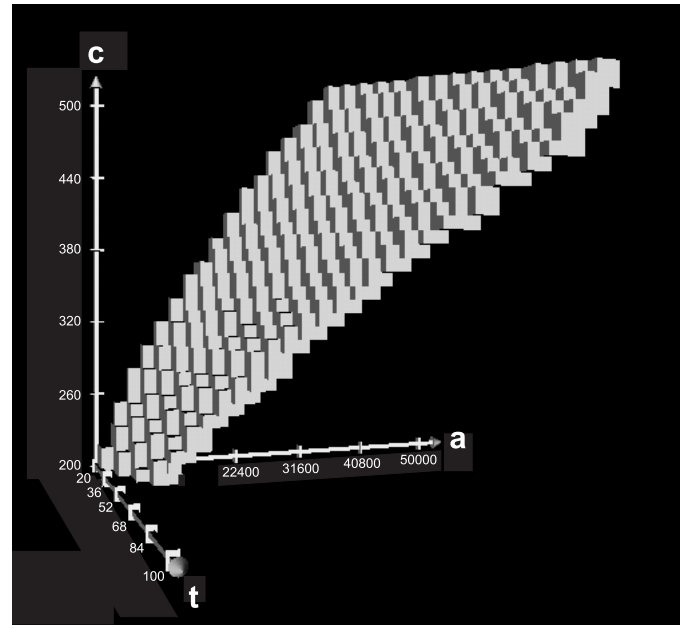

c): total constraint

Figure 7: The total constraint of variables a, $c$ and $t$ is the intersection of all constraints which involve a subset of these variables. In this figure a) and b) are spaces defined by individual constraints which form the space defined by the total constraint $c$ ). 


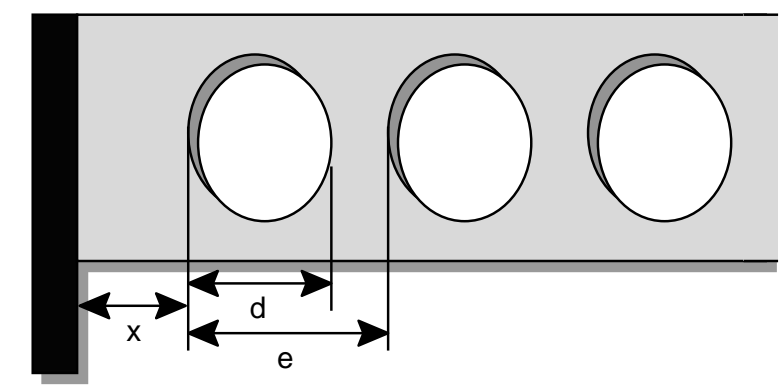

Figure 8: Beam with holes for ventilation ducts. The parameters $\mathrm{d}$, e and $\mathrm{x}$ are involved in constraints of all collaborators.

ventilation engines and avoid excessive energy consumption. The constraints given by the engineer are mostly related to strength and serviceability. Verification of shear and bending moment at several cross-sections is required. In this example, the engineer is the partner that introduces the most of constraints. Many of these constraints are closely related to important geometrical variables such as building height. Traditionally a change in the value of one variable could require a reevaluation of the design by all partners.

For example, if the architect decides to change the beam spacing, the loading carried by beams increases, thereby requiring modifications to cross-sectional characteristics (beam depth, flange size and web thickness) and all associated requirements have to be reverified. Such inter-relationships partly explain the conservative behavior of many structural engineers; they add extra margins of safety in order to absorb most changes without lengthy reverification. Under current situations, this is often economically justifiable. The cost of reverifying a modification to a design that was initially "optimised" - along with the accompanying need to reconsider relevant design decisions as well as the impact of possible omissions when carrying this out - often exceeds the extra cost of the additional margin of safety in the original design. In this situation, the performance of the tools available to engineers have a direct impact on project costs.

Variables associated with this example are given in Table 2 and constraints are listed in Table 3. Constraints are placed in categories according to their functional requirements. The strength constraints are most complicated. Some of the constraints are taken from Swiss building codes [39, 40] while others were fixed by the owner of the building. Engineers are most often the project partners that have been overloaded in the past when too many changes to projects occur and when there is no efficient computational support for managing dependencies between variables. Therefore, of all project tasks, it is expected that this tool will support engineering change-management tasks the most.

\subsection{Analysis of the constraint satisfaction problem}

Examples of interdependencies between partners along with associated variables are given in Figure 9. Although no two partners are independent, some links are stronger than others. For example, there are many shared variables between the engineer and the architect and therefore, many possibilities for conflicting assignments for values of variables. Conversely there is a weak link between the ventilation sub-contractor and the steel fabricator.

SpaceSolver detects a conflict using only arc-consistency methods if $c_{v}$ has a value of 


\begin{tabular}{clcl}
\hline $2 c$ & flange width & $L_{s c}$ & short term live load \\
$a$ & chord height above the hole & $M_{f h}$ & clear floor height \\
$a_{n}$ & lever arm of $N_{1}$ & $M$ & bending moment \\
$A_{1}$ & chord area above the hole & $n_{d}$ & number of ducts \\
$A_{1 w}$ & web area above the hole & $N_{1}$ & force in the chord \\
$b$ & intermediary value & $N_{1 p l}$ & strength of the chord \\
$B_{v}$ & floor volume & $n_{h}$ & number of holes \\
$b_{s}$ & beam spacing & $Q_{s c}$ & short term linear load \\
$c_{v}$ & coefficient of air renewal & $Q_{r}$ & load \\
$c_{n}$ & chord centre of gravity & $S$ & static moment \\
$d_{o}$ & duct thickness with isolation & $t$ & flange thickness \\
$d_{v}$ & ventilation ducts' diameter & $t_{f s}$ & ceiling thickness \\
$d$ & hole diameter & $t_{s}$ & floor surface thickness \\
$e$ & center-to-center hole spacing & $t_{w}$ & web thickness \\
$E$ & Young's modulus & $\tau_{d}$ & shear stress \\
$F_{r}$ & maximal air speed in ducts & $V_{f}$ & air flow in the duct \\
$F_{h}$ & total floor height & $V_{1}$ & shear force \\
$f_{y}$ & elastic strength & $V_{p l 1}$ & shear strength \\
$q$ & linear total load & $V_{2}$ & shear force \\
$G_{r}$ & resistance factor & $V_{p l 2}$ & shear strength \\
$G_{q}$ & load factor & $w_{a}$ & allowable deflection \\
$G_{m}$ & self weight & $w_{l}$ & beam deflection, short term load \\
$h$ & total beam height & $x$ & distance from support to first hole \\
$I_{y}$ & moment of inertia & $y_{1}$ & coordinate of the first hole \\
$L$ & beam span & $y_{2}$ & coordinate ofthe second hole \\
& & $Z_{1}$ & plastic modulus \\
\hline & &
\end{tabular}

Table 2: Definition of parameters for the steel framed building

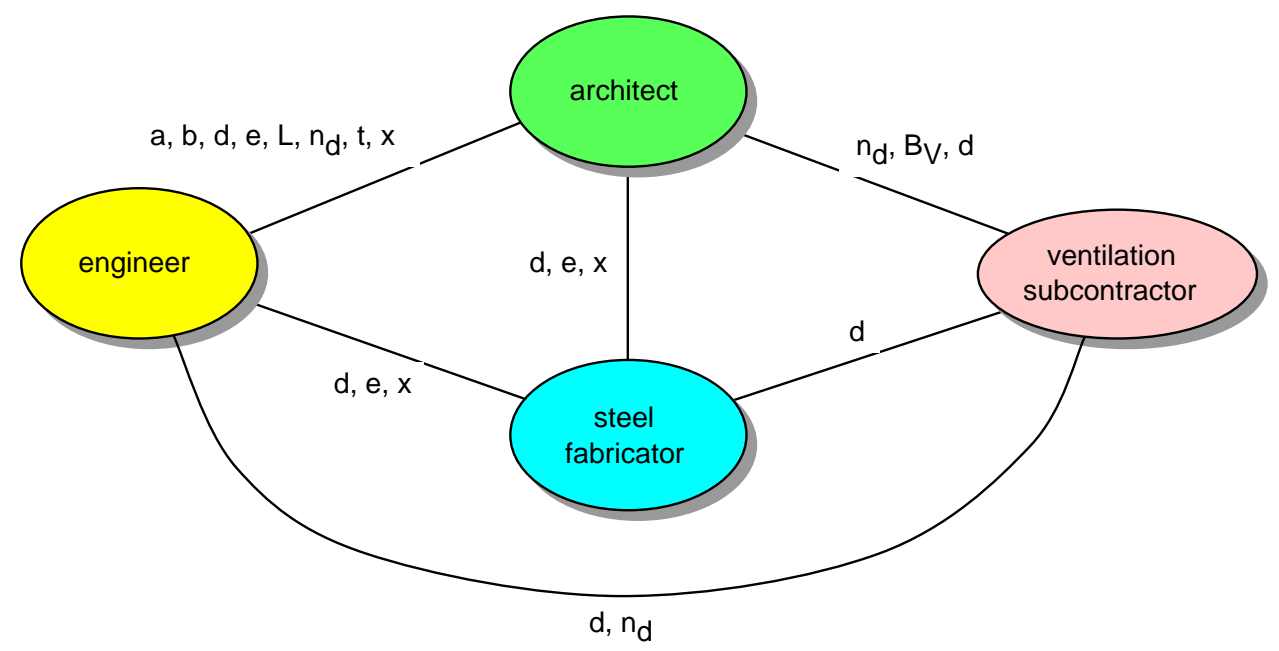

Figure 9: Examples of interdependencies between partners and values for variables for Application 1 . 
Geometry

$$
\begin{aligned}
L & =2 x+n_{h} d+\left(n_{h}-1\right) e \\
F_{H} & =M_{F H}+h+t_{s}+t_{f s} \\
B_{V} & =F_{H} A_{b} \\
h & =2 a+d \\
h & =b+t \\
n_{d} & \leq n_{h} \\
3 & \leq n_{h}
\end{aligned}
$$

$\underline{\text { Manufacturing }}$

$$
\begin{aligned}
1.5 d & <x \\
2.5 d & <e \\
d & <0.75 h
\end{aligned}
$$

\section{$\underline{\text { Ventilation Constraints }}$}

$$
\begin{aligned}
d_{v}+d_{o} & <d \\
c_{V} B_{v} & <V_{f} \\
V_{f} & =n_{d} F_{r} \pi d_{v}{ }^{2} / 4
\end{aligned}
$$

$\underline{\text { Strength Constraints }}$

$$
\begin{aligned}
N_{1} & <N_{1 p l} / G_{r} \\
N_{1} & =M / a_{N} \\
M & =q L^{2} / 8 \\
q & =\left(1.3 G_{m}+G_{q} Q_{r}\right) b_{s} \\
a_{N} & =S / A_{1} \\
S & =2 c t b+t_{w}(a-t)(b-a) \\
\frac{N_{1}}{A_{1}} & <\frac{\sqrt{f_{y}^{2}-3 \tau_{d}^{2}}}{G_{r}}-\frac{V_{1} d}{4 Z_{1}} \\
N_{1 p l} & =f_{y} A_{1}
\end{aligned}
$$

Strength Constraints (cont.)

$$
\begin{aligned}
& A_{1}=2 c t+t_{w}(a-t) \\
& \tau_{d}=V_{1} / A_{1 w} \\
& A_{1 w}=(a-t / 2) t_{w} \\
& V_{1}=\frac{q L}{4}-\frac{q}{2}\left(x+\frac{d}{2}\right) \\
& V_{1}<V_{1 p l} / G_{r} \\
& V_{2}<V_{2 p l} / G_{r} \\
& V_{2}=\frac{q\left(y_{2}{ }^{2}-y_{1}{ }^{2}+L e\right)}{2 a_{N}} \\
& y_{1}=x+d / 2 \\
& y_{2}=y_{1}+e \\
& V_{1 p l}=f_{y} A_{1 w} \sqrt{3} / 3 \\
& V_{2 p l}=f_{y} A_{2} \sqrt{3} / 3 \\
& A_{2}=(e-d) t_{w} \\
& 11<\frac{a-t / 2}{t_{w}} \\
& w_{a}>1.2 w_{l} \\
& w_{l}=\frac{5}{384} \frac{q_{s c} L^{4}}{E I_{y}} \\
& q_{s c}=L_{s c} b_{s} \\
& I_{y}=c t b^{2}+\frac{t_{w}(a-t)(b-a)^{2}}{2} \\
& w_{a}=\frac{1}{350} L \\
& 2 c t<t_{w}(a-t) \\
& c_{n}=\frac{A_{1}}{2 t_{w}} \\
& Z_{1}=\frac{c_{n}{ }^{2} t_{w}}{2}+\frac{\left(a-c_{n}-t\right)^{2} t_{w}}{2} \\
& +2\left(a-c_{n}-\frac{t}{2}\right) c t
\end{aligned}
$$

Table 3: Constraints in the above table provide an example of requirements for using castellated beams in a steel-framed computer-building. 
more than 0.0015 and only two ventilation ducts are used. A few seconds are enough to detect this conflict; either more ducts are needed or such a high air exchange rate cannot be reached.

In the absence of requirements on the order of instantiating values to variables, it is advantageous to develop negotiation plan where variable values influencing the work of many collaborators are fixed first. Such decisions make subsequent variable assignments more independent and therefore opportunities to perform negotiation efforts in parallel become available. Figure 10 shows that when the value for hole diameter, $d$, is fixed, the problem separates into two easier problems. After a decision regarding the number of ventilation ducts, $n_{d}$, there are three easier problems.

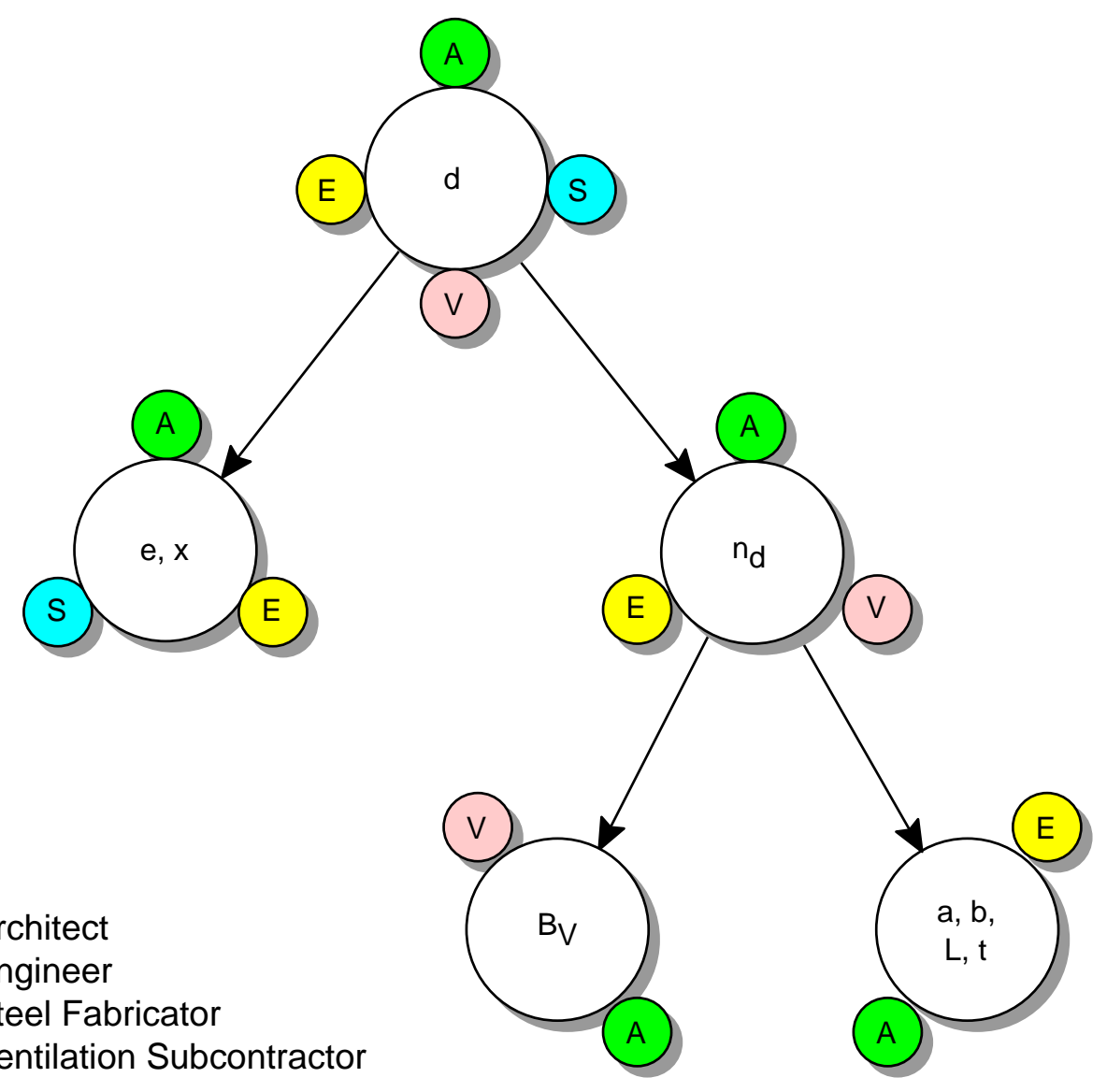

Figure 10: In order to minimize conflicts and backtracking during negotiation, parameters influencing the work of many collaborators can be fixed first. This yields a partial order for the agenda during negotiation, indicated with arrows in this picture.

An example of a projection of the solution space onto three variables is given in Figure 11. Here allowable values of beam spacing, beam depth and air renewal quality are given. If any value is chosen for these three variables in the space shown, there exists acceptable values for all other variables. In order to simplify this example and maintain a reasonable execution time, several variables (for example, load and resistance factors, loading, yield stress, ultimate strengths and Young's modulus) were assigned constant values. The resulting CSP had 17 variables that were not taken to be constant and took 150 minutes to execute 
on a Sun UltraSparc II.

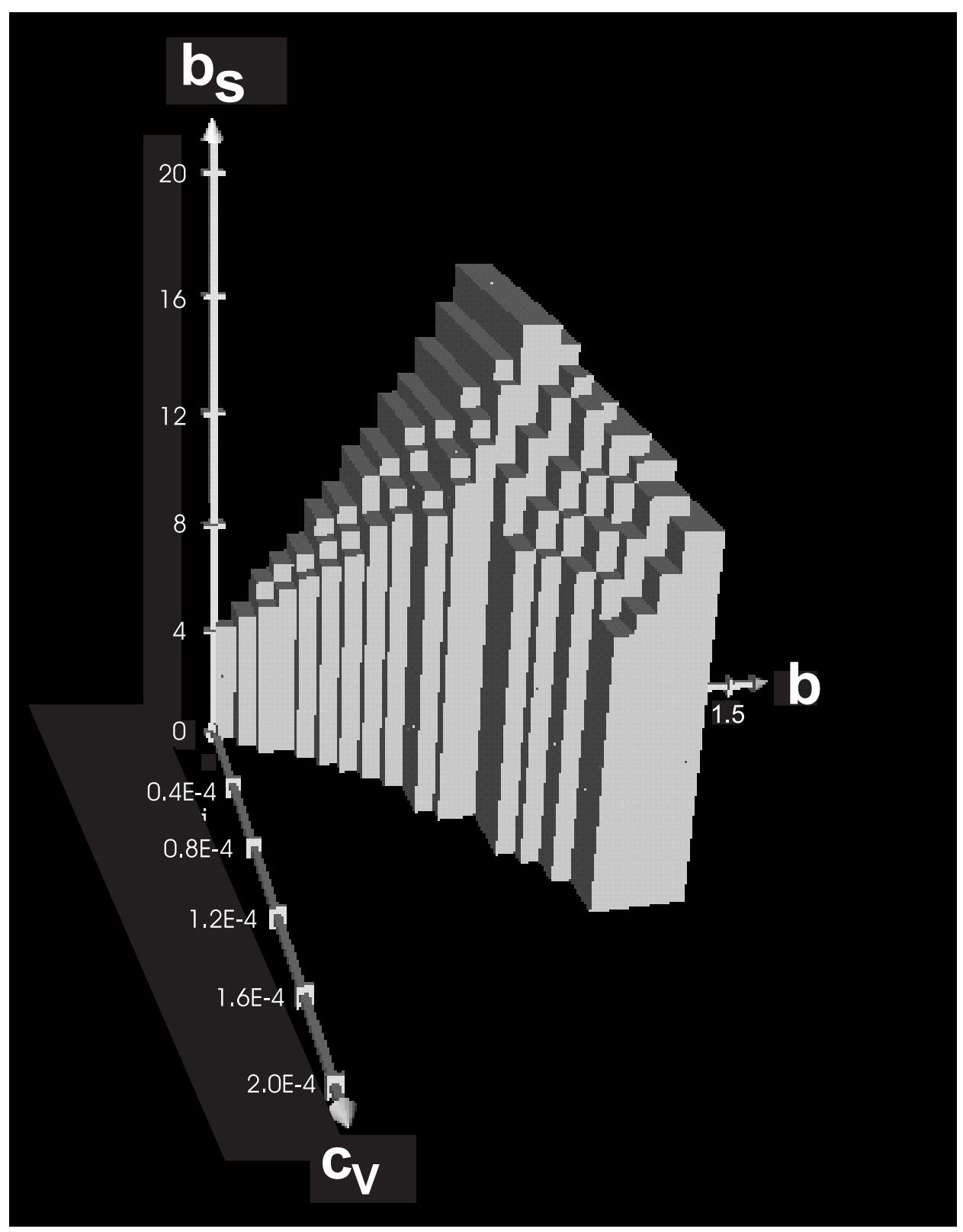

Figure 11: The globally consistent solution space for beam spacing, beam depth and the quality of air renewal for Application 1.

\subsection{Application 2: Vibrations in a stacked gym building}

This example is taken from a building built in Bienne, Switzerland. In the city centre there was a project to build two triple gymnastic halls. Due to limited space, the one gym was placed on top of the other. This lead to special constraints related to vibration of the floor beams of the upper gym. In addition, the owners had special requirements related to the position of these beams since they are used to fix equipment that is used in the lower gym. 
Local building laws include a height restriction for this zone and in addition, the building is situated on a near-surface aquifer. If foundations were placed below the water table, pumping and waterproofing costs would have been unacceptable. Therefore, a solution for squeezing two gyms between the building height limit and the water table, while avoiding vibration problems and meeting owner requirements, had to be found.
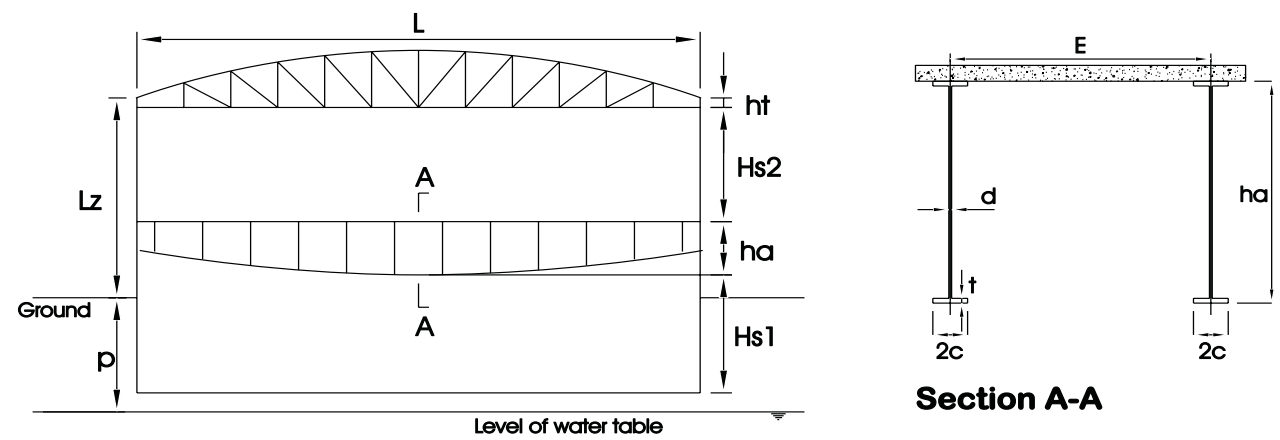

Section A-A

Figure 12: Application 2. Two gyms are stacked for reasons of space. This leads to constraints on vibrations in the upper floor and potential conflicts with height limitations and excessive waterproofing costs

Figure 12 includes a drawing of the building cross section and a section showing the floor beams. Examples of the variables are given in Table 4 and constraints are shown in Table 5. The network of partners and parameters is shown in Figure 13. An example of a solution space for this problem is given in Figure 3 for beam height, modal stiffness and flange width. In this figure, parts a and b took only a few seconds to calculate, whereas the space in part c required approximately two hours on a Ultra Sparc II. The problem involves 16 variables and 12 constraints. The calculation is performed for the complete network. The solution space projection for any three variables in the constraint network is easily plotted on axes such as those in Figure 3.

\begin{tabular}{clcl}
\hline $2 c$ & flange width & $L_{z}$ & height restriction \\
$d$ & web thickness & $P$ & footing depth \\
$E$ & beam spacing & $q^{*}$ & self weight of concrete slab \\
$h_{a}$ & beam height & $R_{f}$ & dynamic factor \\
$H_{s 1}$ & lower gymnastic room height & $S$ & safety coefficient \\
$H_{s 2}$ & upper gymnastic room height & $t$ & flange thickness \\
$h_{t}$ & upper beam minimal height & $\omega_{n}$ & eigenfrequency \\
$m^{*}$ & modal mass & & \\
$k^{*}$ & modal stiffness & & \\
\hline
\end{tabular}

Table 4: Definition of parameters for the stacked gyms building, Application 2.

\section{Limitations and Discussion}

Our implementation of constraint-based support for collaboration in construction currently has several limitations. Determination of global consistency for complex projects where sev- 


$$
\begin{aligned}
q_{\text {concrete }} & =200 \cdot E^{0.6} & \frac{h_{a} \cdot d}{500} & \geq \frac{\sqrt{3}}{235} \cdot L \cdot E^{1.6} \cdot R_{f} \cdot S \\
m^{*} & =q_{\text {concrete }} \cdot E \cdot \frac{L}{2} & \frac{t c \cdot h_{a}}{500} & \geq \frac{250}{235} \cdot L \cdot E^{1.6} \cdot R_{f} \cdot S \\
k^{*} & =\frac{E_{\text {steel }}}{10^{6} \cdot L^{3}} \cdot\left(3.37 \cdot h_{a}^{3} \cdot d\right. & L_{z}+P & \geq H_{s 1}+H_{s 2}+h_{a}+h_{t} \\
& \left.+42.84 \cdot h_{a}^{2} \cdot t c\right) & 4.5 \cdot t & \leq c \leq 8.5 \cdot t \\
\omega_{n} & =\frac{k^{*}}{m^{*}} & H_{s 1} & \geq 5.5, H_{s 2} \geq 5.5 \\
\omega_{n} & \geq 8 \cdot 2 \pi & P & \leq 5, E \geq 2.5
\end{aligned}
$$

Table 5: These constraints represent requirements related to vibration, building height, client requirements and foundation depth for Application 2.

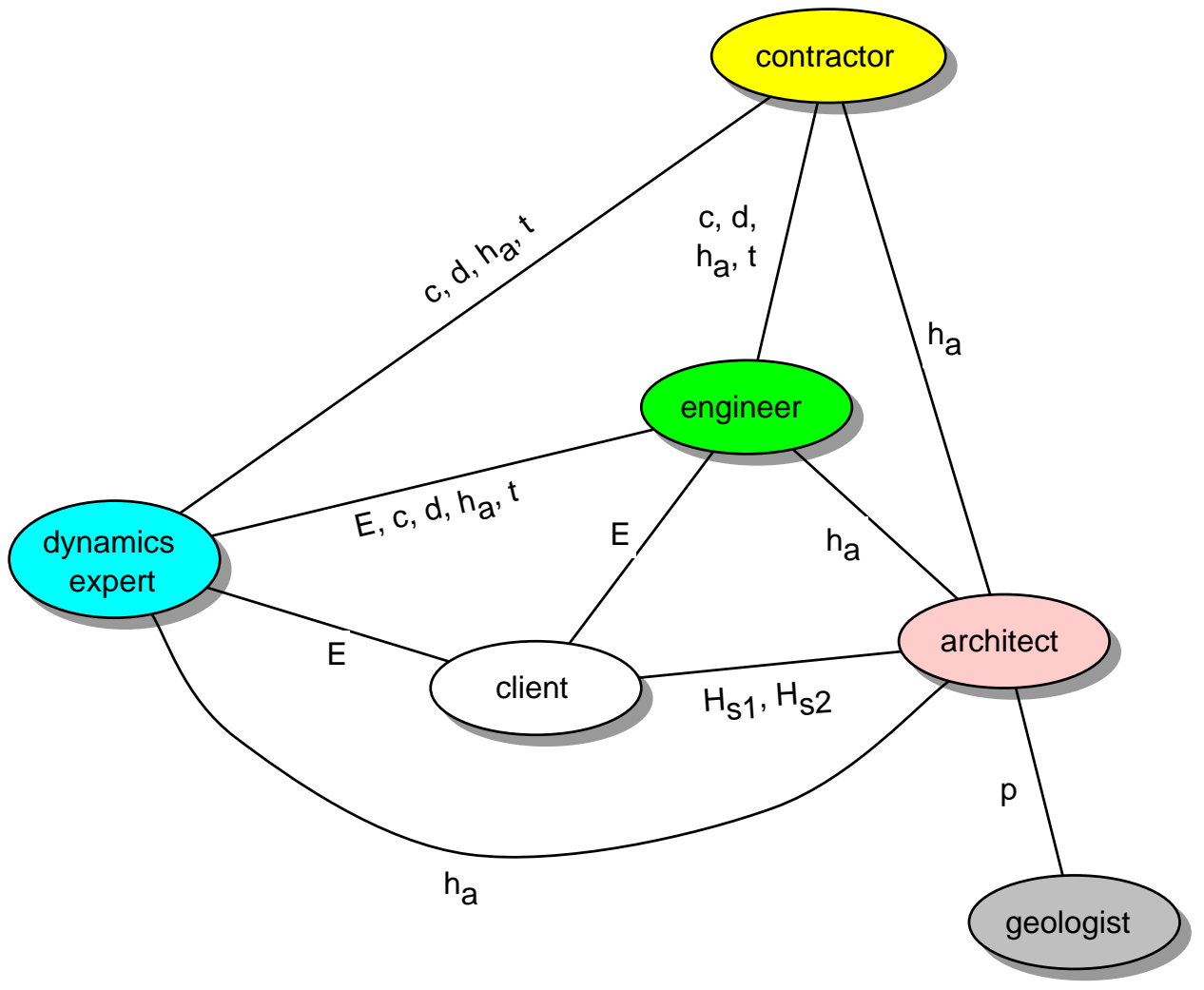

Figure 13: Interdependencies between partners and values for variables for Application 2. 
eral tens of variables are interrelated could require many hours of execution time. Methods for decomposing large constraint networks are currently under study. Partners explore complex problems by making constant distant and non-critical variables in order to simplify calculations. We are currently working on a new version of the software that will allow such interactive exploration.

Disjunctive constraints sets must currently be accommodated one at a time as separate problems. Some work at EPFL has provided interesting results for dynamic constraint satisfaction problems and this may be a promising way to manage multiple constraint sets for collaboration tasks [13]. Currently global management of multiple constraint sets is left to the user.

Another limitation is related to constraint formulation and ease of input. While engineers have little difficulty expressing their requirements in terms of constraints, other project partners are less able to express their requirements in this way. This is due to the characteristics of some functional requirements, traditional work habits and the current user interface. Also, homogenization of terminology will always be a challenging task. Nevertheless, while observing collaboration for full scale construction projects it was noted that even without this tool, partners use constraints without making them explicit and attempt to come to an agreement on terminology. This confirms the hypothesis that when possible, important aspects of a design should be put in mathematical form so that CSP techniques can be activated to maintain project consistency.

CSP algorithms and this implementation provide an opportunity to enhance current proposals for computer supported negotiation. Negotiation cannot be avoided altogether; this contribution is a proposal to improve efficiency of communication and to avoid unnecessary conflict between project partners.

In many practical cases, complete constraint representation is impossible. Our approach does not require constraint sets to be complete. If a constraint is missing from the set, the real solution space may be smaller than calculated. Constraints that cannot be represented algebraically are expressed during negotiation sessions in order to guide selection of the point solution in the solution space. Therefore, constraints should not be thought of as defining feasible solutions but rather, they are a delimitation of what is not possible. CSP techniques thus provide good support for interactive decision making when complete knowledge modelling is not possible. In civil engineering, such situations are hardly exceptional.

In spite of the limitations mentioned in this section, constraint satisfaction techniques provide a useful means for performing least commitment collaboration. This approach often allows for better solutions because fewer uninformed decisions are needed to advance the project. Non-critical decisions are delayed as long as possible. In addition, partners are more open for innovative ideas during negotiation due to more effective support for change management.

A final comment is related to the origins of least commitment collaboration and solution space concepts. Similar strategies have existed for decades as management techniques for business tasks. Computational proposals for design exploration have been proposed for several years, for example [21]. We propose recently developed constraint satisfaction techniques to enrich such activities. Multi-actor collaboration tasks that are carried out over long periods of time (years) stand to benefit the most. 


\section{Conclusions}

Constraints are able to represent important functional requirements in construction. The format of inequalities is a common and natural representation of civil engineering requirements and they are particularly useful for delimiting solution spaces. Explicit constraint modelling also helps identification of an effective negotiation agenda. Constraint satisfaction techniques help avoid useless negotiation and support least commitment decision strategies. Change management is also improved. Therefore, use and manipulation of constraints have much potential to improve productivity during phases of design and construction.

\section{Acknowledgements}

This research was funded as part of a series of projects supported by the Swiss Priority Programme ICS of the Swiss National Science Foundation. We would like to thank G. Schmitt, R. Stouffs, D. Kurmann, B. Tuncer, K.H. Mieusset of ETHZ-CAAD for their collaboration in this project. D. Sam-Haroud of EPFL-LIA performed fundamental constraints research, provided advice on improvement of her algorithms and wrote a base document [33] for Section 3. F. Peña-Mora, D. Sam-Haroud, E. Gelle, K. Shea and B. Raphael as well as the anonymous reviewers are thanked for providing comments on drafts of this paper. Information related to the examples was provided by A. Flückiger, Zwahlen \& Mayr SA, Aigle, Switzerland.

Subject: article.bbl Date: Thu, 8 Oct 1998 09:20:38 +0200 (CEST) From: Ian.Smith@epfl.ch (Ian Smith) To: lottaz@lia.di.epfl.ch

\section{References}

[1] D. Bahler. Mixed quantitative/qualitative method for evaluating compromise solutions to conflicts in collaborative design. Artificial Intelligence for Engineering Design, Analysis and Manufacturing, 9(4):325-336, September 1995.

[2] C. Bessière. Arc-consistency and arc-consistency again. Artificial Intelligence, 65(1):179-190, January 1994.

[3] J. Bowen and D. Bahler. Constraint-based software for concurrent engineering. Computer, 26(1):66-68, January 1993.

[4] M.R. Cutkosky and J.M. Tennenbaum. Collaborative engineering over the internet. Communications of the ACM, 39(9):78-87, September 1996.

[5] Darr T.P. and Birmingham W. "Automated design for concurrent engineering" IEEE Expert 9 (5), (1994) 35-42.

[6] R. Dechter, I. Meiri, and J. Pearl. Temporal constraint networks. Artificial Intelligence, 49(1-3):61-95, January 1991.

[7] E.L. Divita, J.C. Kunz, M.A. Fischer "Collaborative desktop engineering" In AI in Structural Engineering, Lecture Notes in AI. Springer, 1998. 
[8] B.V. Faltings and E.M. Gelle. Local consistency for ternary numeric constraints. In IJCAI-97, volume 1, pages 392-397, 1997.

[9] B.V. Faltings, "Arc-consistency for continuous variables," Artificial Intelligence 65, 1994

[10] E.C. Freuder. A sufficient condition for backtrack-free search. Jrnl. A.C.M., 29(1):2432, January 1982.

[11] R. Fruchter. Conceptual, collaborative building design through shared graphics. IEEE Expert, Intelligent Systems \& their applications, pages 33-41, June 1996.

[12] A. C. Bicharra Garcia and A. Santarosa Vivacqua "The use of active design documents to assist conflict mitigation in concurrent engineering" Advances in Concurrent Engineering, CE96, Technomic, Lancaster PA, pp 65-72, 1996.

[13] E.M. Gelle. On the generation of locally consistent solution spaces in mixed dynamic constraint problems. PhD thesis, Swiss Federal Institute of Technology in Lausanne, Switzerland, 1998.

[14] Hua, K., Faltings, B. and Smith, I. "CADRE : Case-based geometric design" Journal of Artificial Intelligence in Engineering, 10, 1996, pp 171-183.

[15] N. Ivezic and J.H. Garrett "Machine learning for simulation-based support of early collaborative design" Int Journal of Artificial Intelligence for Engineering Design, Analysis and Manufacturing, 12, 1998, pp 123-139

[16] N.G. Jussien and O. Lhomme. Dynamic domain splitting for numeric CSPs. In ECAI98, 1998.

[17] T. Khedro and M. Genesereth. "Progressive negotiation for resolving conflicts among distributed heterogeneous cooperating agents" In 12th National Conference on Artificial Intelligence, AAAI, 1994.

[18] M. Klein. An exception handling approach to enhancing consistency, completeness and correctness in collaborative requirements capture. Journal of Concurrent Engineering Research and Applications, pages 73-80, 1997.

[19] G. Kondrak and P. van Beek. A theoretical evaluation of selected backtracking algorithms. Artificial Intelligence, 89:365-387, 1997.

[20] O. Lhomme. Consistency techniques for numerical CSPs. In IJCAI-93, volume 1, pages 232-238, 1993.

[21] Logan, B. and Smithers, T. "Creativity and design as exploration" Modelling Creativity and Knowledge Based Design, J.S. Gero and M.L. Maher eds, Lawrence Erlbaum 1993, 139-175.

[22] A.K. Mackworth. Consistency in networks of relations. Artificial Intelligence, 8:99-118, 1977. 
[23] R. Mohr and T.C. Henderson. Arc and path consistency revisited. Artificial Intelligence, 28:225-233, 1986.

[24] A. Mokhtar, C. Bédard, and P. Fazio. Information model for managing design changes in a collaborative environment. Journal of computing in civil engineering, 12(2):82-92, April 1998.

[25] D.T. Ndumu and J.M.H. Tah. Agents in computer-assisted collaborative design. In $A I$ in Structural Engineering, Lecture Notes in AI. Springer, 1998.

[26] F. Peña-Mora, D. Siriram and R. Logcher. Design Rationale for Computer-supported Conflict Mitigation Journal of computing in civil engineering, 9(1):57-72, 1995.

[27] F. Peña-Mora A collaborative negotiation methodology for large scale civil engineering and architectural projects. In AI in Structural Engineering, Lecture Notes in AI. Springer, 1998.

[28] F. Peña-Mora and C.Y. Wang. Computer-supported collaborative negotiation methodology. Journal of computing in civil engineering, 12(2):64-81, April 1998.

[29] C. Petrie, H. Jeon, and M.R. Cutkosky. Combining constraint propagation and backtracking for distributed engineering. In Constraints 8 Agents, Collected Papers from the 1997 Workshop, pages 76-82, Menlo Park, CA, 1997. AAAI Press.

[30] C.J. Petrie, T.A. Webster, and M.R. Cutkosky. Using pareto-optimality to coordinate distributed agents. Artificial Intelligence for Engineering Design, Analysis and Manufacturing, 9, pp 269-281, 1995.

[31] Y. Rezgui, G. Cooper and P. Brandon "Information management in a collaborative multiactor environment: the COMMIT approach" Journal of Computing in Civil Engineering, 12, 1998, pp 136-143.

[32] W.M. K. Roddis "Knowledge-based assistants in collaborative engineering" In AI in Structural Engineering, Lecture Notes in AI. Springer, 1998.

[33] D. Sam-Haroud. Constraint consistency techniques for continuous domains. PhD thesis, Swiss Federal Institute of Technology in Lausanne, Thesis No. 1423, Switzerland, 1995.

[34] D. Sam-Haroud and B. Faltings. Consistency techniques for continuous constraints. Constraints, 1(1\&2):85-118, September 1996.

[35] H. Samet, Applications of Spatial Data Structures, Addison-Wesley, Reading, Mass., 1990",

[36] R.J. Scherer. AI methods in concurrent engineering. In AI in Structural Engineering, Lecture Notes in AI. Springer, 1998.

[37] G. Schmitt. A new collaborative design environment for engineers and architects. In AI in Structural Engineering, Lecture Notes in AI. Springer, 1998. 
[38] G. Schmitt, R. Stouffs and M. Engeli, "An architecture for collaborative design support: Models, tools and interfaces" Preprints of IFIP WG 5.2 Workshop on Formal Aspects of Collaborative CAD pp. 125-142, Key Centre of Design Computing, University of Sydney, 1997.

[39] Action sur les structures porteuses. Norme suisse SIA 160, Société Suisse des ingénieurs et architectes (SIA), Zurich, 1989.

[40] Constructions métalliques. Norme suisse SIA 161, Société Suisse des ingénieurs et architectes (SIA), Zurich, 1990.

[41] Smith, I.F.C. Stalker, R. and Lottaz, C. "Creating design objects from cases for interactive spatial composition" Artificial Intelligence in Design'96, Kluwer, 1996, pp 97-116.

[42] D. Sriram, R. Logcher, A. Wong and S. Ahmed "A case study in computer-aided cooperative product development" MIT Technical Report IESL 90-01, 1990.

[43] P. van Beek. On the minimality and decomposability of constraint networks. In William Swartout, editor, Proceedings of the 10th National Conference on Artificial Intelligence, pages 447-452, San Jose, CA, July 1992. MIT Press.

[44] P. van Hentenryck, L. Michel, and F. Benhamou. Newton: Constraint programming over nonlinear constraints. Science of Computer Programming, 30(1-2):83-118, January 1998.

[45] A. Ward, J.K. liker, J.L. Cristiano and D.K. Sobek "The second Toyata Paradox: How delaying decisions can make better cars faster" Sloan Management Review, MIT, Spring 19951998. 